\title{
Über die Verschiebung der Vererbungsrichtung unter dem Einfluß von Kohlensäure.
}

\author{
Von \\ Theodor Hinderer.
}

Mit 8 Tabellen und Taf. III-V.

Eingegangen am 18. August 1913.

Inhaltsübersicht.

I. Einleitung. . . . . . . . . . . . . . . Seite

II. Versuchsbericht. Kreazung von $\frac{\text { Strongylocentrotus } 0}{\text { Sphaerechinus } \$} \ldots \ldots 189$

III. Untersuchung der Larven . . . . . . . . . . . . . . . . . 190

1. Das Skelett der elterlichen Larven. Begriffserklärungen. . . . . 190

2. Die Versehiebung der Vererbungsrichtung unter dem $\operatorname{Einflu} \beta$ von Kohlensäure . . . . . . . . . . . . . . 193

A. Allgemeiner Überblick . . . . . . . . . . . . . 193

B. Zahlenmäßiger Nachweis für die Verschiebung der Vererbungsrichtung. . . . . . . . . . . . 198

a. Die Form der Analarmstïtzen . . . . . . . . 198

b. Die Wurzelzahlen der Analarmstützen . . . . . 199

c. Die Form der analen Scheitelbalken . . . . . . . 200

d. Das Auftreten und die Länge der oralen Scheitelbalken 200

e. Die Länge und das Lüngenverhältnis der analen Scheitel-

balken und Armstützen . . . . . . . . . . 201

C. Erörterung des Ergebnisses . . . . . . . . . . . . . . 204

D. Scheinbare Abweichungen. . . . . . . . . . . . 206

Erklärung der Abbildungen. . . . . . . . . . . . . 209

\section{Einleitung.}

In der Vererbungsforschung sind zwei Hauptrichtungen zu unterscheiden, die vielfach, gegenseitig neue Aufgaben stellend und lösend, in fruchtbarer Verbindung miteinander stehen. Die eine, die sich vorwiegend mit den Nachkommen von Bastarden, besonders mit der *zweiten Bastardgeneration", befaßt, sucht nach Regeln im Auftreten 
bestimmter Vererbungserscheinungen. Sie ist vertreten durch Mended, de Vries, Correns, 'Tschermak, Bateson, Lang, Häcker, DavenPORT, Bauer und viele andere Forscher.

Die andere - die entwicklungsmechanische - Richtung strebt nach der Erkenntnis des Vererbungsmechanismus, oder mit andern Worten: sie erforscht den ursächlichen Zusammenhang zwischen den äußeren und inneren Bedingungen der Entwicklung eines Lebewesens und dem Ergebnis dieser Entwicklung. Mit der Erkenntnis wäre dann höchstwahrscheinlich auch die Möglichkeit gegeben, die Vererbungserscheinungen zu beherrschen.

In dieser Richtung hat außer andern Forschern besonders Herbst Untersuchungen angestellt. Die Frage, zu deren Beantwortung er bis jetzt sieben "Vererbungsstudien * unternommen hat, lautet: * Warum stehen die Nachkommen mit ihren Eigenschaften bisweilen in der Mitte zwischen ihren beiden Eltern, warum neigen sie bisweilen mehr dem einen oder dem andern zu, oder warum gleichen sie mitunter ganz dem einen ihrer Erzeuger, während das Bild des andern unterdruickt zu sein scheint? $(06, \mathrm{I})$.

Die vorliegende Arbeit darf als Fortsetzung der Vererbungsstudien von Herbst gelten. Sie wurde ausgeftihrt auf seine Anregung, mit seiner Unterstitzung und mit dem mir von ihm zur Verflugung gestellten Beobachtungsstoff. Es ist mir ein herzliches Anliegen, Herrn Professor Herbst dafür anch hier meinen wärmsten Dank za sagen.

Aus den Untersuchungen von Boveri, Morgan, Seeliger, SteinBRÜCK und Vernon wußte man, daß aus dem mit Samen von Strongylocentrotus oder Echinus befruchteten Eiern von Sphaerechinus BastardPlutei von sehr verschiedener Vererbungsrichtung hervorgehen können: die Plutei hatten bald mehr oder weniger, bald völlige Ähnlichkeit mit der Larvenform des Vaters oder der Mutter, oder sie konnten als Mittelform zwischen der Gestalt der väterlichen und der muitterlichen Larven bezeichnet werden: A $\mathrm{m} \mathrm{Kalkskelett} \mathrm{der} \mathrm{Larven}$ lassen sich die elterlichen Einflasse nach MaB und Zahl leicht feststellen.

Daraus geht hervor, daß sich die bezeichneten Seeigel hervorragend für Vererbungsstudien eignen; hinzu kommt noch, daß man sie jederzeit leicht erlangen kann, und daß die Keimzellen gegen allerlei ungewöhnliche Behandlung anffallend widerstandsfähig sind.

Meine Untersuchungen sind denn auch, wie die Herbsts, an diesen Seeigeln angestellt, und zwar wurden Eier von Sphaerechinus 
granularis mit Samen von Strongylocentrotus lividus befruchtet. (Diese Kreuzung wollen wir kurz mit $\frac{S t r . O^{T}}{S p h . ~}$ bezeichnen.)

Nach den Angaben von Herbst gebe ich zunächst den

\section{Versuchsbericht 1 ).}

Zu dem in Neapel angestellten Versuch wurde Seewasser benutzt, durch das während einer ganzen Nacht und des ganzen folgenden Vormittags Kohlensäure geleitet worden war. Die Eier wurden, da keines der Weibchen sehr voll war, mehreren SphaerechinusWeibchen entnommen und gemischt. Je ein Teil dieser Eier wurde besonders behandelt:

a) Am 11. April 1910, 12 Uhr 35 Min. nachmittags, warde ein Teil der Eier in eine Mischung von $70 \mathrm{ccm}$ kohlensäurehaltigem und $30 \mathrm{ccm}$ gewöhnlichem Seewasser gebracht. Dieses gemischte Wasser ist 5 Uhr 40 Min. nachmittags abgegossen und durch gewöhnliches Seewasser ersetzt worden. Die Eier lagen also ungefähr 5 Stunden in dem gemischten Wasser.

b) Die Behandlung eines andern Teiles der Eier erfolgte in der gleichen Weise und zur selben Zeit wie bei a; aber die Überfthrung in gewöhnliches Seewasser geschah erst $8 \mathrm{Uhr} 55 \mathrm{Min}$. nachmittags, also etwa 3 Stunden später als bei jenem Teil.

c) Ein dritter Teil der Eier wurde 12 Uhr 35 Min. nachmittags in reines Kohlensäurewasser und $5 \mathrm{Uhr} 35 \mathrm{Min}$., nach 5 Stunden, in gewöhnliches Seewassẹr gebracht.

Bei der Untersuchung am 12. April ergab sich, daß sich die Eier des Teils $c$ am wenigsten und die von a am meisten verändert hatten: dieser enthielt am meisten Eier mit vergrößerten Kernen. In $a$ und $b$ waren häufig Kerne mit bisweilen sehr kleinem Plasmateil abgeschntirt.

Am 12. April, vormittags $9 \mathrm{Uhr}$, wurden die Eier von a, von denen manche sich parthenogenetisch weiter entwickelt hatten, mit Samen von Strongylocentrotus vermischt. Nach einer halben Stunde wurde dann ein Teil der Eier in Sublimateisessig eingelegt, weitere Teile in Zwischenräumen von je einer Viertelstunde bis zu 3 Stunden nach der Befruchtung. Außerdem sind zur vergleichenden Prüfung unbefruchtete, unbehandelte und unbefruchtete, behandelte Eier eingelegt worden.

1) Vgl. hierzn C. Herbst, Vererbungsstudie VIII in Sitzungsberichte der Heidelberger Akademie der Wissenschaften. Math.-nat. Kl. Abt. B. 1913. 
Aus den befruchteten Eiern, die man ihrer Entwicklung tuberlassen hatte, waren neben gewöhnlichen Bastardlarven sehr viele mit mutterwärts verschobener Vererbungsrichtung entstanden. Am 16. April, 8 Uhr 55 Min. nachmittags, wurden die Larven abgetötet, und zwar mit neutralem Formol in Seewasser. Eine gute Färbung der Larvenkerne wurde mit Ammoniakkarmin erzielt, welches das Skelett völlig unversehrt ließ.

\section{Untersuchung der Larven.}

Schon bei verhältnismäßig schwacher Vergrößerung und bei flichtiger Durchsicht erkennt man unter den Larven solche mit großen und solche mit kleinen Kernen. Die großen Kerne selbst sind wieder von verschiedener Größe. Worin das begründet ist, wird später za zeigen sein. In der ersten und zweiten Tabelle habe ich die Ergebnisse der Messungen und Zählungen am Skelett von 50 großkernigen und 50 kleinkernigen Larven nach bestimmten Gesichtspunkten eingetragen. Das Skelett jeder einzelnen Larve ist damit in den fur uns wichtigen Punkten genau geschildert, und es läßt sich durch Vergleich mit den elterlichen Larvenformen feststellen, ob und in welchem Grade eine Verschiebung der Vererbungsrichtung erfolgte. Es ist also zunächst nötig, die Larven von Strongylocentrotus und von Sphaerechinus kennen zu lernen.

\section{Das Skelett der elterlichen Larven.}

Begriffserklärungen.!

Die Larven sind zweiseitig-symmetrisch gebaut. Der Hauptteil, in dem der Darm liegt und welcher Scheitelteil heißt, besitzt Kegelform, und zwar gleicht der von Strongylocentrotus (Fig. 1) einem zugespitzten Kegel, wogegen der von Sphaerechinus (Fig. 20) einem durch eine krumme Fläche abgestumpften Kegel verglichen werden kann.

Von der Grundfläche aus geht auf der Oral- und Analseite je ein Lappen, von denen jeder in zwei Fortsätze oder Arme ausläuft, die von einer aus erhöhten Wimperzellen gebildeten einheitlichen Wimperschnur umsäumt werden. Man nennt die Kalkstlitzen dieser oralen und analen Arme Oral- und Analarmstïtzen. Die Oralarmstuitzen sind ebenso wie die analen Querstäbe bei den beiden Larvenformen einfache Stäbe, kommen also für uns nicht in Betracht. Wichtig für unsere $Z_{w e c k e}$ sind aber die bei den Strongylocentrotus- und Sphaerechinus-Larven sehr verschieden gestalteten Analarmstïtzen. 
Während die Analarmstutze der Strongylocentrotus-Larve ein einfacher gerader Stab ist, wird die von Sphaerechinus von drei (selten vier) gleichlaufenden Stäben gebildet, die durch etwa 15-20 schmale Querbrücken verbunden sind. Wir nennen eine solche Analarmstiitze drei- (oder vier-) kantig.

Die Stutzen des Scheitelteiles heißen Scheitelbalken. Bei der Larve von Strongylocentrotus sind dies in der Fortsetzung der analen Armstützen verlaufende Stäbe mit allmählich anschwellenden freien Enden, meist glatt, doch nicht gerade selten wie mit kleinen Dornen besetzt. Das Skelett des Scheitelteiles der Sphaerechinus-Larve wird als Scheitelkorb bezeichnet. Die analen Scheitelbalken der beiden Seiten sind zum größten Teil gerade oder leicht nach anßen gekrümmt, biegen dann aber unter einem stumpfen Winkel um und vereinigen sich an ihren Enden. Von den oralen Armstntzen gehen die beiden oralen Scheitelbalken aus, die sich je in der Weise verzweigen, daß ein Ast mit dem entsprechenden der Gegenseite und der andere Ast sich mit einem Zweig vom Endstiick des analen Scheitelbalkens der gleichen Seite verbindet. Durch diese Verzweigung entsteht das Gerüst der Scheitelwölbung, der Scheitelrahmen. Die Grenze zwischen dem Scheitelteil und den Armstützen liegt an einer in der Linie: analer Scheitelbalken - anale Armstiitze durch einen sanften Knick bezeichneten Stelle, von der aus auch der anale Querstab abgeht.

Von Bedeutung für diese Untersuchung ist endlich das Längenverhältnis der analen Scheitelbalken und der analen Armstlitzen. Dies ist bei Strongylocentrotus ungefähr wie $1: 1$, bei Sphaerechinus wie $1: 2$.

Der Besprechung der ersten and zweiten Tabelle müssen einige Bemerkungen über die Grundsätze vorausgeschickt werden, die mich bei der Abfassung leiteten.

Beide Tabellen sind in durchaus ubereinstimmender Weise abgefaßt, weil dadurch ein Vergleich leichter gemacht wird. Obwohl also in der ersten Tabelle in Reihe XI und in der zweiten Tabelle in Reihe III keine Einträge zu machen waren, habe ich diese Reihen doch nicht ausgelassen - sie lassen auf den ersten Blick den Unterschied zwischen den entsprechenden Reihen der andern Tabelle erkennen.

In den Reihen VI bis XI sind die Zahlen der Wurzeln der Analarmstützen verzeichnet. Wir betrachteten als •Wurzel * jeden kurzen oder langen Kalkstab, der in der Grenzgegend zwischen dem Scheitel- 
teil und den Fortsätzen seinen Ursprung nimmt und in den Analarm derselben Seite hineingerichtet ist. Die Strongylocentrotus-Larve hat also gewöhnlich Analarmstlitzen mit einer Wurzel, die SphaerechinusLarve solche mit drei (vier) Wurzeln.

Die Reihe V enthält die Zahlen der »gleichlaufenden, unverbundenen Stäbe in den Analarmen*. Dazu gentigt wohl die Bemerknng, daß mindestens zwei gleichlaufende Stäbe bis ans Ende des Armes unverbunden sein mußten, um hier vermerkt zu werden. Stummel warden besonders darum nicht bericksichtigt, weil bei größerer Länge vielleicht eine Querverbindung mit einem andern Stab hätte auftreten können; wie z. B. Fig. 7 zeigt, kommen manchmal erst in größerem Abstand vom Ursprung Querverbindungen vor.

Wenn zwei solche Stäbe an ihrem Ende verschmelzen, so haben wir eine Stlitze mit zwei Wurzeln vor uns; trennen sich die Stäbe aber wieder, so handelt es sich um eine Stutze mit zwei Wurzeln und einer Querverbindung (Fig. 5).

Einer Erlänterung bedarf schließlich noch die Reihe VII: "Analarmstuitzen mit einer Wurzel und mit Gabelung oder Ansatz zur Gitterbildung «. „Gabelung * ist ohne weiteres verständlich. "Ansatz zar Gitterbildung * kommt nur bei einem Pluteus (Nummer 8 der ersten Tabelle, Fig. 8) vor: die Gabeläste vereinigen sich am Ende. In der zweiten Tabelle sind die Plutei 6, 20, 30, 43 und 47 mit einwurzeligen Analarmsttitzen aufgefuhrt. Man kann zwar bei ihnen nicht mehr von *Ansatz* zur Gitterbildung reden, da sie, wie ein Blick in die IV. Reihe zeigt, 3-14 Querverbindungen zwischen den Gabelästen oder zwischen dem Hauptstab und einem gleichlaufenden, wurzellosen Seitenast besitzen. Es liegt hier also eine eigentliche Gitterbildung vor, doch konnten die Larven nicht gut anders eingereiht werden, eben weil die Stiitzen nur eine Wurzel haben. Dem Umstand, daß die Larven im Grundplan zweiseitig-symmetrisch gebaut sind, jedoch meist, namentlich bei den Bastardlarven, im Skelett mehr oder weniger asymmetrische Formen und Größenverbältnisse zeigen, habe ich in den Tabellen so Rechnung getragen, daß ich die an der linken Skeletthälfte festgestellten Zahlen auf der linken, die der rechten Hälfte anf der rechten Seite der Reihe eintrug. (Dabei nahm ich, ohne eine tiefere Absicht als die, alle Larven einer genau gleichen Betrachtung zu unterziehen, immer die Lage der Larve so an, daß die Oralseite mir zugekehrt war.) 
2. Die Verschiebung der Vererbungsrichtung unter dem Einfluß von Kohlensäure.

\section{A. Ein allgemeiner Überblick}

uiber die beiden Tabellen zeigt uns schon die Unterschiede im Skelett der großkernigen und kleinkernigen Larven. Statt »großkernige * und skleinkernige « Larven können wir auch sagen: Larven, bei denen die Behandlung der Eier mit Kohlensäure eine deutlich sichtbare Kernvergrößerung bewirkt hat, und Larven, bei denen eine solche Wirkung nicht zu sehen ist. Wir stellen nun an Hand der Tabellen zuerst allgemein, dann so genau wie möglich, den Einfluß der Kohlensäure auf die Gestaltung der Bastardlarven fest.

Vergleichen wir die Reihen II der beiden Tabellen, so wird sofort deutlich, daß die Zahl der Analarmstittzen mit Querverbindungen bei den großkernigen Larven viel größer ist als bei den kleinkernigen, und wenn wir dazu noch die Reihen IV halten, so finden wir, daß auch die Zahl der Querverbindungen in der zweiten Tabelle bedeutend höher ist als in der ersten. Stützen mit nur einer Querverbindung weist die III. Reihe der zweiten Tabelle im Gegensatz zu jener der ersten überhaupt nicht auf. Kurz: Gitter sind bei den Larven mit großen Kernen viel häufiger und viel besser ausgebildet, als bei denen mit kleinen Kernen.

Weiter zeigt die erste Tabelle in der XVI. Reihe (Bemerkungen) keinen einzigen Fall, wo die Analarmstütze dreikantig zu nennen wäre; die zweite Tabelle dagegen führt eine erhebliche Zahl solcher Stutzen auf, ja sogar eine vierkantige Sttitze finden wir in ihr.

Eine Zusammenfassung der Reihen VI bis XI ergibt für die großkernigen Larven eine weit größere Gesamtzahl der Wurzeln, als für die kleinkernigen.

Die Endigungen der analen Scheitelbalken sind bei den Larven mit kleinen Kernen durchschnittlich einfacher als bei den großkernigen Plutei nnd besitzen nie, wie immerhin mehrere von diesen, die kennzeichnende Gestalt der analen Scheitelbalken von Sphaerechinus-Larven.

Orale Scheitelbalken kommen bei den kleinkernigen Larven nicht entfernt so häufig und anch meist nicht in solch bedeutender Länge vor, wie bei den großkernigen.

Endlich zeigen die Reihen XII und XIII der beiden Tabellen beztiglich des Längenverhältnisses der analen Scheitelbalken und analen Armstutzen wichtige Unterschiede: bei den Larven mit großen 
1. Tabelle. Platei mit

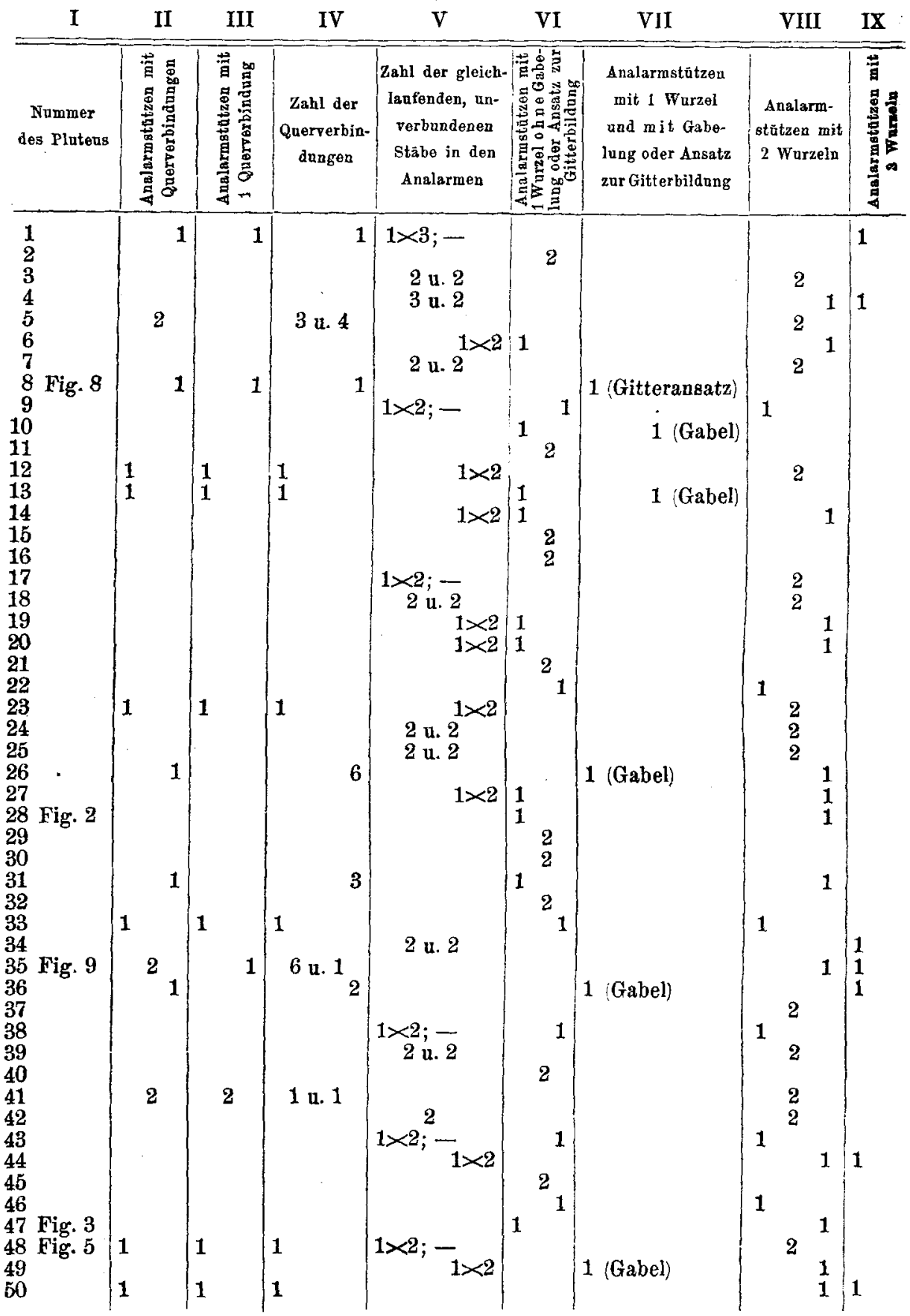

1) Die Länge wurde in Teilstrichen des Okularmikrometers von Zeiss Ok. II mit 
kleinen Kernen.

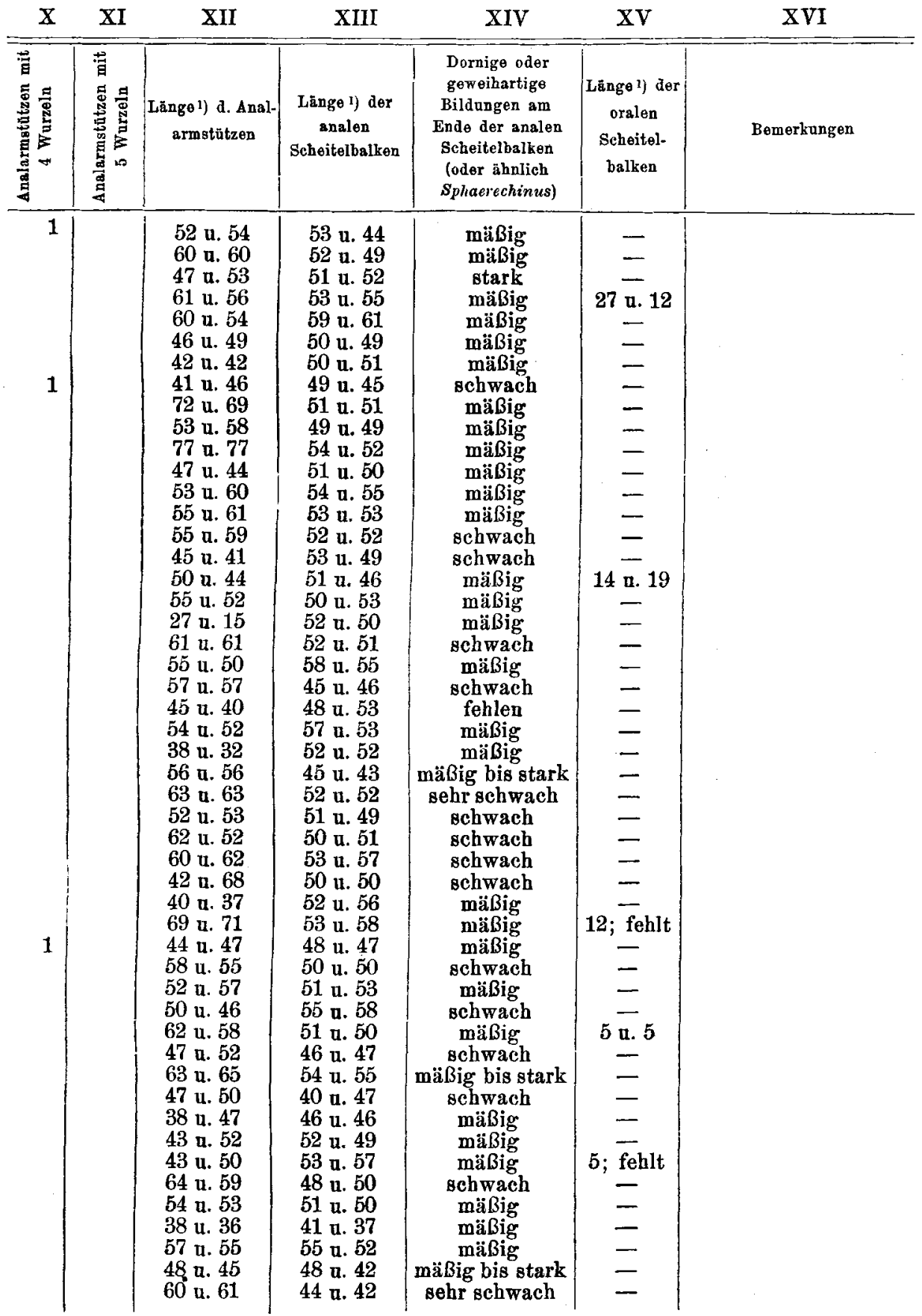

SEIBert 0 bj. 3 bei eingeschobenem Tubrs gemessen. 
2. Tabelle. Platei mit

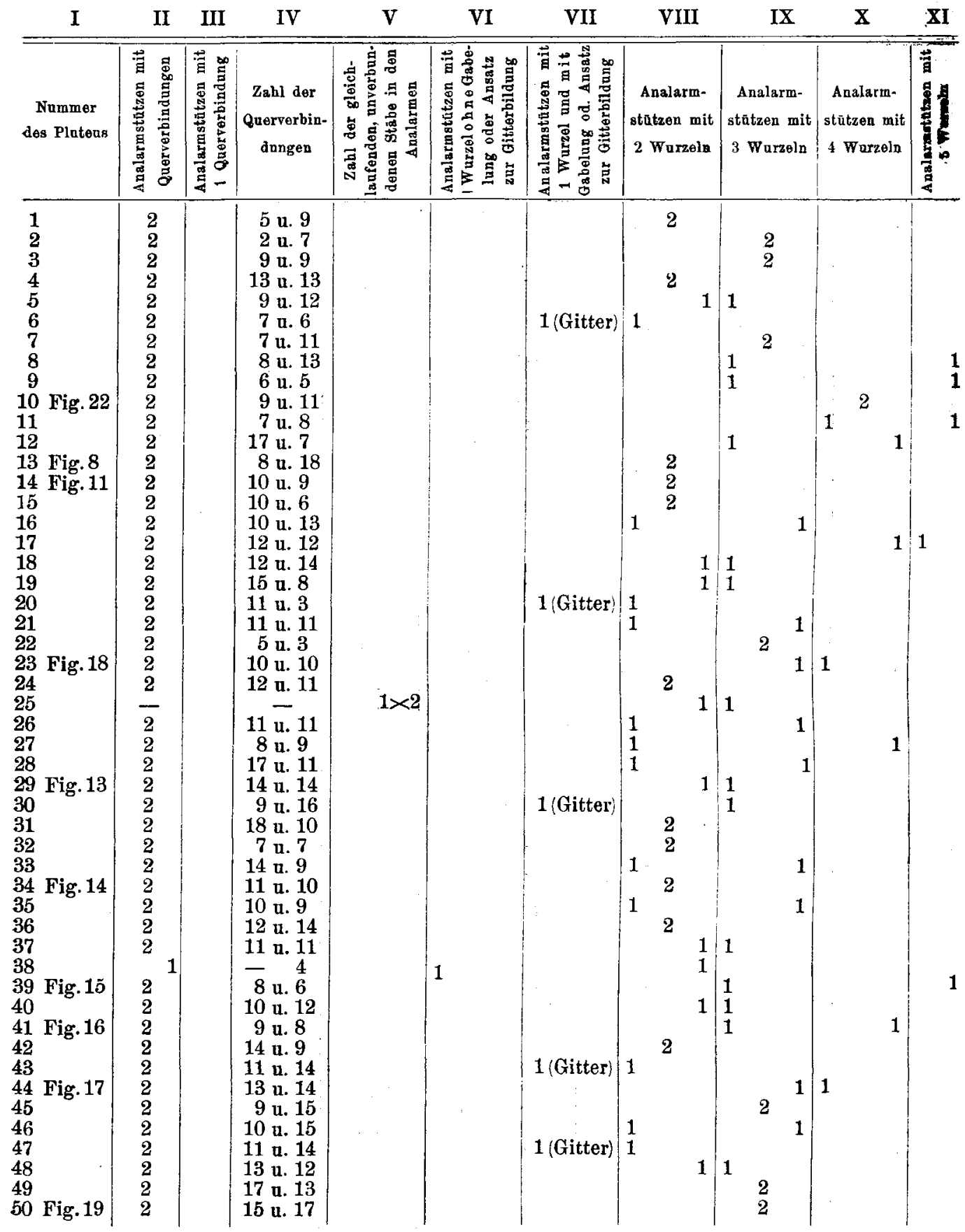

1) Die Länge wurde in Teilstrichen des Okularmikrometers von Zeiss Ok. II mit SErBert 


\section{großen Kernen.}

\begin{tabular}{|c|c|c|c|c|}
\hline XII & XIII & XIV & XV & XVI \\
\hline 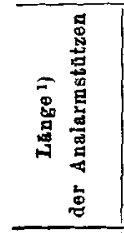 & 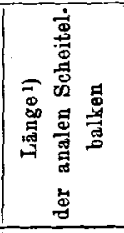 & $\begin{array}{c}\text { Dornige oder geweih- } \\
\text { artige Bildungen am Ende } \\
\text { d. analen Scheitelbalken } \\
\text { Oder âlnlich Sphaer. } \\
\text { echivus }\end{array}$ & 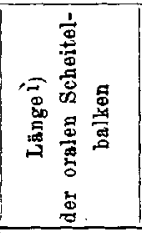 & Bemerkangen \\
\hline u. 61 & 45 il. 50 & mäßig & 10 u. 7 & \\
\hline 62 u. 62 & 49 น. 45 & sehr stark & 9 u. 5 & linke Analarmstütze z. Teil dreikantig \\
\hline 69 บ. 66 & 43 и. 43 & mäßig & 7 แ. 8 & linke Analarmstütze dreikantig \\
\hline $\begin{array}{l}66 \text { u. } 66 \\
72 \text { a. } 78\end{array}$ & $\begin{array}{l}44 \text { u. } 40 \\
45 \text { u. } 50\end{array}$ & stark & 29: Fehlt & \\
\hline $\begin{array}{l}\text { a. } 78 \\
\text { a. } 72\end{array}$ & $\begin{array}{l}45 \text { u. } 50 \\
47 \text { u. } 44\end{array}$ & & $\begin{array}{l}29 ; \text { fehlt } \\
\text { fehlt: } 16\end{array}$ & linke Analarmstütze dreikantig \\
\hline u. 62 & 44 u. 44 & mäßig & - & rechte Analarmstötze dreikantig \\
\hline 5 u. 65 & 50 น. 50 & mäBig & 12 u. 12 & Analarmstūtzen dreikantig; Oralarmstūtzen nư Lānge 12 \\
\hline 5 u. 54 & 45 น. 50 & stark & 32 ; fehlt & rechte Analarmstūtze dreikantig \\
\hline 39 น. 40 & 33 u. 34 & ähnlich Sphaerech. & 32 n. 19 & linke Analarmstütze 4 kantig; rechte Analarmstatze 3 kantig \\
\hline 54 u. 53 & 44 a. 50 & stark & 31; fehlt & Analarmstützen teilweige 3 kantig \\
\hline 68 и. 53 & 50 u. 46 & mäßig & 20 u. 8 & Analarmstützen 3 kantig \\
\hline $\begin{array}{l}65 \text { น. } 64 \\
70 \text { น. } 67\end{array}$ & $\begin{array}{l}40 \text { u. } 42 \\
49 \text { u. } 52\end{array}$ & $\begin{array}{l}\text { mäßig bis stark } \\
\text { stark }\end{array}$ & - & 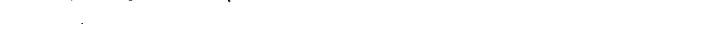 \\
\hline 63 u. 63 & 40 u. 43 & ähnlich Sphaerech. & $9 \overline{\text { u. } 20}$ & . \\
\hline 72 u. 76 & 39 น. 38 & ähnlich Sphaerech. & 9 u. 41 & rechte Analarmstūtze 3 kantig \\
\hline 58 u. 58 & 49 u. 47 & stark & 29 ; fehlt & Analarmstützen 3 kantig \\
\hline 57 น.. 63 & 48 u. 49 & stark & 30 ; fehlt & \\
\hline 77 u. 72 & 46 u. 46 & stark & 5 ; fehlt & linke Analiarmstütze 3 kantig \\
\hline 60 u. 59 & 47 u. 45 & stark & fehlt; 11 & \\
\hline 54 u. 57 & 48 u. 49 & mäbig. & 22 น. 13 & rechte Analarmstütze 3 kantig \\
\hline 50 u. 57 & 40 u. 50 & mäßig & - & \\
\hline 57 u. 68 & 52 u. 52 & mäßig & 36 u. 37 & linke Analarmstütre z. Toil 3 kantig \\
\hline 62 u. 65 & 42 и. 39 & mäßig & 10 ; fehlt & \\
\hline 68 u. 64 & $\tilde{0} 0$ u. 46 & mïßig & 9 u. 16 & linke Analarmstūtze besitat 1 lang, u. 2 ziemlich lange Wurzeln \\
\hline 61 น. 59 & 47 น. 50 & mïBig & 5 u. 7 & \\
\hline 45 u. 59 & 42 u. 43 & mäßig bis stark & fehlt; 19 & rechte Analarmstütze 3 kantig \\
\hline 73 u. 73 & 44 1. 45 & mäßig & 11 u. 30 & ebenso \\
\hline 73 น. 73 & 42 и. 49 & stark & 7 u. 32 & \\
\hline 73 u. 71 & 51 u. 51 & mäßig & 8 u. 30 & linke Analarmstũtze auf kurze Strecke 3 kantig \\
\hline 80 เฉ. 74 & 44 a. 43 & mäßig & 15 и. 18 & \\
\hline 71 u. 72 & 45 น. 45 & mäßig & fehlt; 32 & linke Analarmstūtze zur Hălfte 3 kantig \\
\hline 62 u. 66 & 49 u. 45 & mäßig & fehlt; 5 & rechte Analarmstätze 3 kantig, linke Analarmst. am Ende 3 kantig \\
\hline 74 น. 70 & 50 u. 47 & stark & 22 u. 31 & 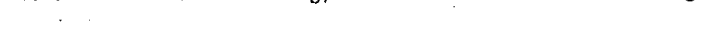 \\
\hline 56 u. 55 & 35 น. 44 & sehr stark & 14; fehlt & \\
\hline 63 u. 44 & 44 ก. 44 & mäBig bis stark & 35 u. 29 & \\
\hline 62 & 45 u. 44 & ähnlich Sphaerech. & 6 ; fehlt & linke Analarmstūtz 93 kantig, rechte Analarmst. am Ende 3 spitzig \\
\hline 59 & 46 и. 45 & mäß̂́ig & 17 u. 24 & \\
\hline a. 49 & 48 a. 49 & schwach & 7; feblt & rechte Analarmstūtze 3 kantig \\
\hline 59 u. 58 & 45 u. 47 & mäßig & 10 ; fehlt & linke Analarmstütze. 3 kantig \\
\hline 59 & 42 น. 38 & schwach bis mäßig & 24 u. 24 & linke Analarmst. 3 kantig, rechte Analarmst. stellenweise 3 kantig \\
\hline 76 u. 65 & 44 u. 44 & mäßig & fehlt; 44 & \\
\hline 64 & 43 u. 43 & stark & fehlt; 37 & \\
\hline 69 u. 65 & 48 n. 47 & stark & 5 n. 34 & linke Analarrastütze zar Bălfte 3 kantig, reehte A. z. T. 3 kantig \\
\hline 35 u. 71 & 49 u. 52 & mäßig & 14 u. 25 & Analarmstintzen 3 kantig \\
\hline 71 u. 75 & 42 u. 41 & ähnlich Sphaerech. & 26 น. 35 & rechte Analarmstūtze 3 kantig \\
\hline 31 u. 63 & 46 u. 44 & sehr stark & fehlt; 34 & \\
\hline $\begin{array}{l}64 \text { u. } 57 \\
65 \text { v. } 65\end{array}$ & 46 11. 47 & $\stackrel{\text { stark }}{\text { mark }}$ & 31 ; fehlt & linke A, teilweise 3 kantig, rechte A. 3 kant., am Ende 4 Spitzen \\
\hline $\begin{array}{l}50 \text { n. } 60 \\
36 \text { u. } 63\end{array}$ & $\begin{array}{l}51 \text { u. } 52 \\
35 \text { a. } 35\end{array}$ & $\begin{array}{l}\text { mäBig bis stark } \\
\text { ähnlich Sphaerech. }\end{array}$ & $26 \overline{\mathrm{u}} .28$ & $\begin{array}{l}\text { von d. linken A. das letzte Viertel } 3 \text { kantig; d. linke A. } 3 \text { kantig } \\
\text { Analarmstützen } 3 \text { kantig }\end{array}$ \\
\hline & & & & \\
\hline
\end{tabular}

Obj. 3 bei eingeschobenem Tabus gemessen. 
Kernen tritt das Verhältnis $1: 2$, wie es bei Sphaerechinus ist, genau oder doch mit deutlicher Annäherang viel häufiger auf, als bei den kleinkernigen Larven, bei denen es mehr wie $1: 1$, also wie bei Strongylocentrotus ist. Bei diesen kommt es sogar einigemal vor, daß die analen Scheitelbalken länger sind als die Analarmstuttzen, was wir bei den großkernigen Larven nie finden.

Aus dieser allgemeinen Übersicht sehen wir schon einigermaßen deutlich, daß es sich immer um ein Mehr oder Weniger handelt, und zwar um ein Mehr oder Weniger der muitterlichen Artmerkmale. Das Mehr kommt den großkernigen Larven zu. Wir können also jetzt schon sagen: die Larven mit großen Kernen zeigen mehr Anklänge an die Mutter, als die mit kleinen Kernen. Mit andern Worten: in den Fällen, in denen durch die Kohlensäure eine Kernvergrößerung bewirkt wurde, ist die Vererbungsrichtung im Vergleich zu den Larven mit augenscheinlich unveränderten Kernen nach der mütterlichen Seite verschoben. Dieses Ergebnis soll im folgenden noch deutlicher gemacht werden.

B. Zahlenmäßiger Nachweis für die Verschiebung der Vererbungsrichtung.

a. Die Form der Analarmstützen.

Aus der ersten und zweiten Tabelle erhalten wir die Zahlen der dritten Tabelle.

3. Tabelle.

\begin{tabular}{|c|c|c|c|c|c|c|c|}
\hline $\begin{array}{l}\text { Kern- } \\
\text { grổe }\end{array}$ & 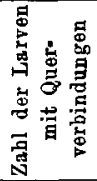 & $\begin{array}{l}\text { Zahl d. Anal- } \\
\text { armstatzen } \\
\text { mit } 1 \text { Quer- } \\
\text { verbindnng }\end{array}$ & 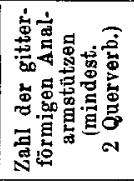 & $\begin{array}{c}\text { Zahl der } \\
\text { streckenweise od. } \\
\text { ganz } 3 \text { bantigen } \\
\text { Analarmstützen }\end{array}$ & 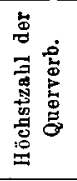 & $\begin{array}{l}\text { Durchschnitts- } \\
\text { zahl der } \\
\text { Querverb. einer } \\
\text { Analarmstütze }\end{array}$ & 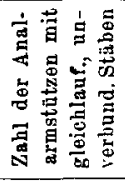 \\
\hline klein & 14 & 11 & 7 & 0 & 6 & nicht ganz $1 / 2$ & 31 \\
\hline groß & 49 & 0 & 97 & $\begin{array}{c}41 \\
\text { (dav. } 1 \text { vierk. })\end{array}$ & 18 & 10 & 1 \\
\hline
\end{tabular}

Diese Zahlen reden eine deutliche Sprache. Man kann wohl sagen, daB die Analarmstitzen der kleinkernigen Larven rechten Bastardcharakter tragen. Der mütterliche Einfluß äußert sich bei ihnen hauptsächlich darin, daß bei einem Drittel der Stïtzen gleichlaufende, unverbundene Stäbe auftreten und (selten) Querverbindungen vorkommen, und daß, wie wir gleich zeigen werden, bei etwas mehr als der Hälfte die Zahl der Wurzeln vermehrt wurde. Demgegen- 
über ist bei den Larven mit großen Kernen das stärkere Hervortreten mütterlicher Kennzeichen mit aller wünschenswerten Deutlichkeit wahrzunehmen. Die sich gegentiberstehenden Zahlen 14 und 49, 7 und 97,0 und 41,6 und 18, $1 / 2$ und 10 bezeichnen so erhebliche Unterschiede, daß sie jeden.Zweifel an der Verschiebung der Vererbungsrichtung nach der miutterlichen Seite ausschließen.

Stellen wir

b. die Wurzelzahlen der Analarmstützen

einander gegentiber.

4. Tabelle.

\begin{tabular}{|c|c|c|c|c|c|c|c|}
\hline $\begin{array}{l}\text { Kern- } \\
\text { größe }\end{array}$ & $\begin{array}{c}\text { Zahl der Anal- } \\
\text { armstítzen mit } \\
1 \text { Wurzel, ohne } \\
\text { Gabelnng oder } \\
\text { Ansatz zur } \\
\text { Gitterbildung }\end{array}$ & $\begin{array}{c}\text { Zahl der Anal- } \\
\text { armstätzen mit } \\
1 \text { wurzel a. mit } \\
\text { Gabelung oder } \\
\text { Ansatz zur } \\
\text { Gitterbildung }\end{array}$ & 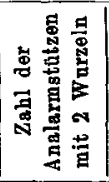 & 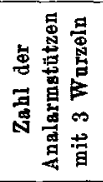 & 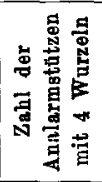 & 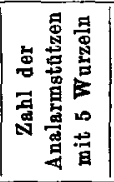 & $\begin{array}{c}\text { Durchsehnitts- } \\
\text { zahl d. Wurzeln } \\
\text { einer } \\
\text { Analarmstatze }\end{array}$ \\
\hline klein & 36 & $\begin{array}{c}6 \text { (davon } 1 \\
\text { Gitteransatz) }\end{array}$ & 48 & 7 & 3 & 0 & $(1,7)$ \\
\hline groß & 1 & 5 (Gitter) & 43 & 37 & 9 & 5 & $(2,6)$ \\
\hline
\end{tabular}

Unter den 50 kleinkernigen Larven waren 36 Analarmstitzen zu zählen, die durchans denen von Strongylocentrotus glichen; unter den großkernigen war nur eine solche aus einem Stab bestehende Analarmstiitze. Die füf gitterförmigen, einwurzeligen Sttitzen lassen gegen die sechs Stiutzen, die nur einmal Gitteransatz und flunfmal Gabeln zeigten, einen deutlich verstärkten Einflaß des mütterlichen Erbanteils erkennen.

Ziemlich gleich für die Larven mit großen und kleinen Kernen ist die Zahl der Analarmstitzen mit zwei Wurzeln. Der große Unterschied liegt hier, wie wir sahen, in dem Vorhandensein oder Fehlen von Querverbindungen, ganz abgesehen davon, daß eine der beiden Wurzeln bei den kleinkernigen Larven in sieben Fällen stummelförmig war, was bei den großkernigen nie vorkam.

Recht in die Augen springend ist der Unterschied, wenn wir die Zahlen der drei-, vier- und fünfwurzeligen Stützen vergleichen, und wenn wir noch dazu halten, daß bei den großkernigen Larven oft alle drei Wurzeln die ganze Länge des Analarms erreichten, bei den kleinkernigen dagegen nur zweimal und ohne Querverbindungen zu besitzen.

Durchschittlich ist die Zahl der Wurzeln bei den großkernigen Larven um eins größer als bei den kleinkernigen. 
c. Die Form der analen Scheitelbalken.

Ich habe bei der vergleichenden Beschreibung der Larvenskelette von Strongylocentrotus und Sphaerechinus schon bemerkt, daß die analen Scheitelbalken der Strongylocentrotus-Larve meist einfache, glatte Stäbe mit angeschwollenen, freien Enden sind, daß aber anch manchmal kleine dornige Bildungen an ihnen auftreten. Bei unsern Bastardlarven bilden solche und andere ungewöhnliche Formen die Regel. Nur bei einer einzigen Larve fehlten sie ganz; bei fast allen andern Pluteis traten sie stärker hervor, als an StrongylocentrotusLarven, was wohl sicher dem mütterlichen Erbanteil zugeschrieben werden darf. Über die Formen an den Enden der analen Scheitelbalken und ihre gradmäßige Beurteilung geben die Abbildungen (Fig. 1-20) genügenden Aufschluß. Hier soll nun gezeigt werden, daß in der Ausbildang dieser Formen bei den klein- und den großkernigen Larven merkliche Unterschiede vorhanden waren. (Die beiden analen Scheitelbalken habe ich bezuiglich ihrer Form zusammen als e in Bild betrachtet und so in den Tabellen aufgeführt.)

5. Tabelle.

\begin{tabular}{l|c|c|c|c}
\hline \hline Kern- & $\begin{array}{c}\text { Zahl d. Larven ohne } \\
\text { oder mit schwach } \\
\text { auggebildet. Sonder- } \\
\text { formen an den analen } \\
\text { Scheitelbalken }\end{array}$ & $\begin{array}{c}\text { Zahl der Larven mit } \\
\text { mäßig oder mäßig } \\
\text { bis stark ausgebild. } \\
\text { Sonderformen an den } \\
\text { anal. Scheitelbalken }\end{array}$ & $\begin{array}{c}\text { Zahl der Larven mit } \\
\text { stark oder sehr stark } \\
\text { ausgebildet. Sonder- } \\
\text { formen an den analen } \\
\text { Scheitelbalken }\end{array}$ & $\begin{array}{c}\text { Zahl der Larven mit } \\
\text { anal. Scheitelbalken } \\
\text { ăhnlich denen von } \\
\text { Sphaerechinus }\end{array}$ \\
\hline \begin{tabular}{c|c|c|c|c} 
klein \\
groß
\end{tabular} & 17 & 32 & 1 & 0 \\
2 & 25 & 17 & 6
\end{tabular}

Man sieht aus der 5. Tabelle, daß nur zwei der großkernigen Larven in der Form der analen Scheitelbalken dem StrongylocentrotusPluteus sehr nahe stehen. Nahezu die Hälfte $(17+6)$ der Larven mit großen Kernen zeigt stark oder sehr stark ausgebildete Sonderformen oder gar die Gestalt der Sphaerechinus-Scheitelbalken. Es stehen sich hier die Zahlen 17 und 1, 6 und 0 gegentiber - ein sprechender Beweis ftur das stärkere Hervortreten der mutterlichen Merkmale bei den Larven mit großen Kernen.

In der folgenden Tabelle vergleichen wir

d. das Auftreten und die Länge der oralen Scheitelbalken, wobei wir auch die stummelförmigen Anlagen mitzählen.

Der Unterschied zwischen den kleinen und großkernigen Larven ist aus diesen Zahlen mit eindringlicher Deutlichkeit zu erkennen. 
6. Tabelle.

\begin{tabular}{l|c|c|c|c}
\hline Kern- & $\begin{array}{c}\text { Zahl der Larven } \\
\text { mit oralen } \\
\text { Scŏße }\end{array}$ & $\begin{array}{c}\text { Zahl der Larven } \\
\text { mit 1 oralen } \\
\text { Seheitelbalken }\end{array}$ & $\begin{array}{c}\text { Zahl der Larven } \\
\text { mit 2 oralen } \\
\text { Scheitelbalken }\end{array}$ & $\begin{array}{c}\text { Größte Länge } \\
\text { der oralen } \\
\text { Scheitelbalken }\end{array}$ \\
\hline klein & 5 & 2 & 3 & 27 \\
groß & 44 & 20 & 24 & 44
\end{tabular}

Es bleibt nur noch hinzuzufügen, $d a ß$ die oralen Scheitelbalken bei den Larven mit großen Kernen in seltenen Fällen sich verzweigten, wie die der Sphaerechinus-Plutens, was aber bei den kleinkernigen Larven nie zu sehen war. Nur in zwei Fällen erreichte der orale Scheitelbalken das Endstlick des analen Scheitelbalkens, aber auch nur bei Larven mit großen Kernen. Die unter dem kernvergrößernden Einfluß der Kohlensäure erfolgte Verschiebung der Vererbungsrichtung nach der mutterlichen Seite tritt also auch an den oralen Scheitelbalken klar hervor.

Die siebente und achte Tabelle geben uns schließlich Auskunft uber

e. đie Länge und das Längenverhältnis der analen Scheitelbalken und Armstützen.

Die 7. Tabelle gibt uns Aufsehluß über das Verhältnis der Länge von Analscheitelbalken und Analarmstlitzen bei je 100 Skeletthälften der klein- und großkernigen Larven. Beim Strongylocentrotus-Pluteus verhält sich, wie schon oben ausgeführt wurde, die Länge der

7. Tabelle.

\begin{tabular}{|c|c|c|c|c|c|}
\hline Kern- & $\begin{array}{c}\text { Zahl der Skelett- } \\
\text { hälften mit dem } \\
\text { Verhăltnis }\end{array}$ & $\begin{array}{c}\text { Zabl der Skelett- } \\
\text { hālften mit dem } \\
\text { Verhältnis }\end{array}$ & $\begin{array}{c}\text { Zahl der Skelett- } \\
\text { hälften mit derm } \\
\text { Verhãltnis }\end{array}$ & $\begin{array}{c}\text { Zahl der Skelett. } \\
\text { hălften mit dem } \\
\text { Verhăltnis }\end{array}$ & $\begin{array}{c}\text { Zahl der Skelett- } \\
\text { hälften mit dem } \\
\text { Verhāltnis }\end{array}$ \\
\hline grổe & $\underline{\text { Sch. }}=1$ & Seh. $=3$ & Sch. $=2$ & $\underline{S c h}_{-}=1$ & Sch. $>1$ \\
\hline$\therefore$ & $\overline{A .}=\overline{1}$ & A. $=\overline{4}$ & $\overline{\mathbf{A} .}=\mathbf{3}$ & $\bar{A} . \overline{2}$ & A. $>1$ \\
\hline klein & 53 & 11 & 5 & 0 & 31 \\
\hline groß & 23 & 28 & 41 & 8 & 0 \\
\hline
\end{tabular}

analen Scheitelbalken zur Länge der analen Armstützen wie 1:1 (wir wollen das kurz so bezeichnen: $\frac{\text { Sch. }}{A .}=\frac{1}{1}$ ); bei Sphaerechinus ist $\frac{\text { Sch. }}{A .}=\frac{1}{2} . \quad$ Es ist nun interessant zu sehen, welche Werte dieses Ver- 
hältnis $\frac{\text { Scheitelbalkenlänge }}{\text { Analarmlänge }}$ bei den Larven mit großen und kleinen Kernen annimmt. Um eine recht deutliche Anschauung vom Unterschied dieser Längenverhältnisse bei den zweierlei Bastardformen zu geben, habe ich die 200 Skeletthälften nach vier Verhältnisstufen geordnet: $\frac{\text { Sch. }}{\mathrm{A} .}=\frac{1}{1},=\frac{3}{4},=\frac{2}{3},=\frac{1}{2}$. Bei den kleinkernigen Larven fand ich eine große Zahl von Skeletthälften, bei denen $\frac{\text { Sch. }}{A .}>\frac{1}{1}$ war. Ich habe aber in der betreffenden Spalte nur die aufgefuhrt, bei denen die Scheitelbalken mindestens um zwei Teilstriche länger waren als die Analarmstïtzen; wo der Untersehied geringer war, betrachtete ich $\frac{\text { Sch. }}{\text { A. }}=\frac{1}{1} . \quad$ Dem Verhältnis $\frac{\text { Sch. }}{\text { A. }}=\frac{1}{1}$ zählte ich noch folgende Brtiche zu: $\frac{5}{6}, \frac{6}{7}, \frac{11}{13}, \frac{13}{15}, \frac{7}{8}, \frac{8}{9}, \frac{9}{10}, \frac{10}{11}, \frac{11}{12}$ und $\frac{12}{13}$. Zu dem Wert $\frac{3}{4}$ rechnete ich außerdem $\frac{7}{9}, \frac{10}{13}, \frac{4}{5}, \frac{13}{16}$ und $\frac{9}{11} ;$ zu $\frac{2}{3}$ die Werte $\frac{3}{5}, \frac{5}{8}, \frac{9}{13}, \frac{7}{10}$ und $\frac{5}{7}$; zu $\frac{1}{2}$ noch $\frac{8}{15}, \frac{4}{7}$ und $\frac{7}{12}$.

Wir sehen, daß etwas mehr als die Hälfte der den kleinkernigen und nicht ganz ein Viertel der den großkernigen Larven zugehörigen Skeletthälften das Strongylocentrotus-Verhältnis: $\frac{\text { Sch. }}{\mathbf{A} .}=\frac{1}{1}$ genau oder annähernd besitzt. Jene zeigen also vielmehr als diese das Längenverhältnis des Vaters. Das Verhältnis $\frac{\text { Sch. }}{\mathrm{A} \text {. }}=\frac{3}{4}$ weisen nur elf Skeletthälften der Larven mit kleinen Kernen auf - das stärkere Hervortreten der mütterlichen Merkmale bei den großkernigen Larven wird hier schon klar: die Zahl 28 steht jenen 11 gegenüber. Am deutlichsten zeigen die Tatsache der Verschiebung der Vererbungsrichtung die dritte und vierte Spalte: der Wert $\frac{2}{3}$ wird nur von fünf Skeletthälften der kleinkernigen Larven erreicht, dagegen von $41 \mathrm{der}$ großkernigen Plutei. Endlich war nur bei Larven mit großen Kernen das Verhältnis $\frac{\text { Sch. }}{\mathrm{A} .}=\frac{1}{2}$ (wie bei Sphaerechinus) zu finden, wie umgekehrt $\frac{\text { Sch. }}{\text { A. }}>\frac{1}{1}$ nur bei kleinkernigen Larven auftrat.

Weiteren Aufschluß über das Längenverhältnis und die Längen 
von Analscheitelbalken und Analarmstiitzen soll die folgende achte Tabelle geben.

8. Tabelle.

\begin{tabular}{|c|c|c|c|c|c|c|c|}
\hline $\begin{array}{l}\text { Kern- } \\
\text { grō ße }\end{array}$ & 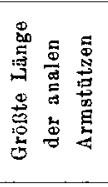 & 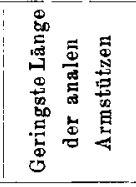 & 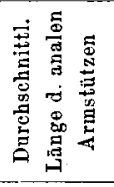 & 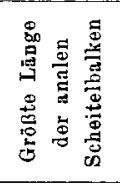 & 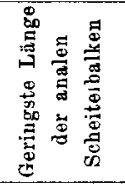 & 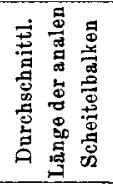 & \begin{tabular}{|c|} 
Durchschnittl. \\
Verbāitnis der \\
Länge d. analen \\
Scheitelbalken \\
zurLänged. anal. \\
Armstützen \\
\end{tabular} \\
\hline klein & 77 & $(15)$ ) 32 & 52,6 & 59 & 40 & 50,6 & fast $\frac{1}{1}$ \\
\hline groß & 80 & $(39 ; 2) 44$ & 63,3 & 52 & $33)^{2}, 35$ & 45,3 & $\frac{5}{7}$ \\
\hline
\end{tabular}

Fassen wir zunächst die Angaben uber den Durchschnittswert des

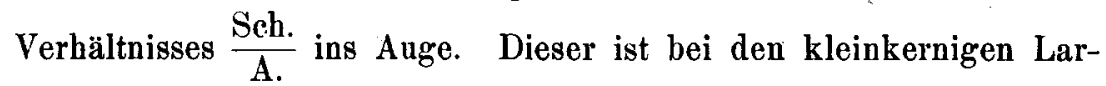
ven fast $=\frac{1}{1}$, bei den Larven mit großen Kernen $=\frac{5}{7}$. In welcher Weise ist nun dieses Durchschnittsverbältnis $\frac{5}{7}$ oder der tatsächlich öfters anftretende Wert $\frac{1}{2}$ - diese mutterwärts gerichtete Verschiebung - bei den großkernigen Larven erreicht? Von vornherein wären drei Fälle möglich:

1) Die Analarmstiltzen werden durchschnittlich länger, als die der kleinkernigen Larven, während die analen Scheitelbalken die gleiche durchschnittliche Länge besitzen.

2) Die analen Scheitelbalken werden bei den großkernigen Larven durchschnittlich kürzer, als bei den kleinkernigen, aber die analen Armstitzen haben bei beiden Larvensorten durchschnittlich dieselbe Länge.

3) Die Analarmstitzen werden durchschnittlich länger und die analen Scheitelbalken durchschnittlich kürzer, als sie bei den kleinkernigen Larven sind.

Dieser dritte Fall wäre zweifellos der beachtenswerteste. Man könnte dann von einem Wachstumsausgleich zwischen Armstab- und

1) Neben diese von allen andern weit abstehende Zahl setze ich die nächstkleinste Angabe der Länge einer offenbar gut (Querverbindungen!) ausgebildeten Analarmstiutze (Plntens 25).

2) Maß einer Zwerglarve (Pluteus 10; daneben die Angabe einer Ganzlarve.

Archir f. EntwickIungsmechanik. XXXVIII. 
Scheitelbalkenlänge reden; die Armstäbe mlissen nicht so lang und die Scheitelbalken nicht so kurz werden, damit eine Annäherung an das Verhältnis $\frac{\text { Sch. }}{A .}=\frac{1}{2}$ oder gar dessen reine Ausbildung erzielt wird, wenn Armstab- und Scheitelbalkenlänge, statt jede allein, sich beide in der bezeichneten Weise gleichzeitig ändern. Wie die Durchsicht der achten Tabelle ergibt, ist bei den großkernigen Larven dieser Ausgleich zur Tatsache geworden. Nicht nur sind die Angaben der größten und kleinsten Längen der Analarmstïtzen bei den Larven mit großen Kernen höher, als bei denen mit kleinen Kernen, nicht nur sind auch die größten und kleinsten Längen der Analscheitelbalken bei jenen geringer, als bei diesen, sondern - und das fällt mehr ins Gewicht - die durchschnittliche Länge der Analarmstützen ist größer, und die durchschnittliche Länge der analen Scheitelbalken ist bei den großkernigen Larven kleiner, als bei den kleinkernigen. Die durchschnittliche Länge der analen Armstützen ist von derjenigen der kleinkernigen Larven mit 52,6 Teilstrichen auf 63,3 der großkernigen, also um fast 11 Teilstriche, gestiegen, während die mittlere Länge der analen Scheitelbalken von 50,6 Teilstrichen bei den Larven mit kleinen Kernen auf 45,3, also um etwa 5 , bei den großkernigen Larven sank. Wir sehen daraus auch, daß sich die Armstablänge um einen größeren Betrag erhöhte, als die Scheitelbalkenlänge sich verringerte. Die Annäherung des

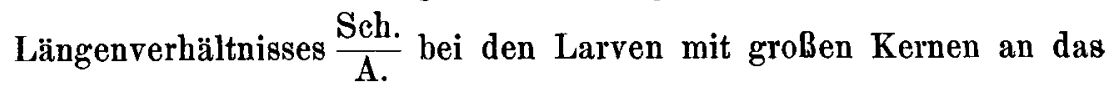
Verhältnis $\frac{1}{2}$ vollzog sich also mehr durch Veränderung der Analarmstützen- als der Scheitelbalkenlänge.

Ich glaube, damit die unter dem Einfluß der Kohlensäure erfolgte Verschiebung der Vererbungsrichtung nach der mütterlichen Seite hinreichend genau und ausführlich bewiesen zu baben. Die Skelette der Larven, die aus Eiern hervorgingen, deren Kern vor der Befruchtung durch die Einwirkung von Kohlensäure vergrößert worden war, wiesen in nicht zu mißdeutender Weise viel mehr Anklänge an die Larve von Sphaerechinus auf, als die Plutei, die sich aus Eiern mit unvergrößerten Kernen entwickelten.

\section{Erörterung des Ergebnisses.}

Dieses Ergebnis könnte nicht in Frage gezogen werden durch seltene Fälle, wo man sagen müßte: diese beiden Larven haben 
ziemlich gleich auggebildete Skelette - aber die eine Larve besitzt große, die andere kleine Kerne; oder: diese beiden Larven sind sehr verschieden im Bau des Skeletts, die eine hat mehr väterliche, die andere mehr muitterliche Skelettmerkmale - und trotzdem haben beide gleich große Kerne. Derartige Merkwürdigkeiten könnte man, wenn man sich nicht mit der Scheinerklärung zufrieden geben mag, daß das eben "zufällig * so sei, vermutungsweise auf die Verschiedenheit der einzelnen Keimzellen zurückführen - es ist denkbar, wenn auch zunächst nicht erklärbar, daß in manchen Eiern der mütterliche oder väterliche Erbanteil anf irgendeine Weise ganz oder zum Teil unwirksam wird, und daß dann der andere Erbanteil entsprechend in den Vordergrund tritt. Eine solche Verschiedenheit lernten wir ja bei unsern Versuchseiern kennen, die zudem aus mehreren und wohl auch etwas verschiedenen Weibchen stammten: bei einem Teil der Eier wurde der Kern durch die Einwirkung der Kohlensäure vergrößert, bei einem andern nicht, ein dritter Teil entwickelte sich parthenogenetisch - das deutet doch darauf hin, daß die Eier, obwohl ohne sichtbare Unterscheidungszeichen, sich irgendwie unterschieden. Auch bei Bastardlarven, deren Eier keiner besonderen Behandlung unterzogen wurden, sind die Unterschiede in der Gestalt des Skeletts oft recht groB: mehr vaterähnliche treten neben mehr mutterähnlichen auf. Es wäre also durchaus nicht verwunderlich, wenn auch unter den Larven dieses Versuchs sich solche großkernige fänden, deren Skelett weniger mütterliche Anklänge als die übrigen zeigte und so den kleinkernigen Larven ähnlich wäre, die etwas mehr mutterliche Merkmale aufwiesen als die andern.

Wir wollen aber doch die beiden ersten Tabellen noch daraufhin durchsehen, ob es uns jetzt möglich wäre, aus der Beschreibung des Skeletts auf die Kerngröße zu schließen, oder ob wir bei mancher Larve im Zweifel wären, welche Kerngröße wir ihr, nach dem Skelett zu urteilen, zuschreiben dürften.

Wenn wir die zweite Tabelle tuberblicken, in der die Skelette der großkernigen Larven beschrieben sind, so sehen wir, daß Querverbindungen nur bei drei Analarmstutzen fehlen, und zwar bei Pluteus 25 an beiden und bei Pluteus 38 nur bei einer Analarmstuitze, daß aber bei den andern Pluteis schon die hohe Zahl der Querverbindungen gegenuiber der niederen bei den kleinkernigen Larven uns über den Zweifel hinwegheben würde, welche Kerngröße wir bei ihnen annehmen dïrften, besonders wenn wir noch die Zahl der Wurzeln, das Vorhandensein und die Länge der oralen Scheitelbalken, das Verhältnis 
$\frac{\text { Sch. }}{\text { A. }}$ und anderes hinzunehmen. Wären wir non wohl versucht, die Larren 25 und 38 als kleinkernig zu bezeichnen? Ich glaube kaum. Denn der Pluteus 25 hat zwar keine Querverbindungen, aber dafür andere Merkmale, die auf einen stärkeren mütterlichen Einfluß hinweisen, als wir ihn sonst bei kleinkernigen Larven finden: er besitzt zwei orale Scheitelbalken, das Verhältnis $\frac{\text { Sch. }}{A .}$ der beiden Skeletthälften ist $=\frac{3}{4}$, die linke Analarmstütze bat drei Wurzeln, von denen eine sehr lang und die beiden andern ziemlich lang (nicht nur Stummel) sind. Die Larve 38 ist durch zwei orale Scheitelbalken von ansehnlicher Länge ausgezeichnet, weiter besitzt die rechte Skeletthälfte das Verbältnis $\frac{\text { Sch. }}{\mathrm{A} .}=\frac{3}{4}$ und die rechte Analarmstütze vier Querverbindungen.

Wie würden wir uns aber bei den Pluteis $4,5,17,26,35,38$, 44 der ersten Tabelle entscheiden? Ich greife die beiden Plutei 4 und 35 herans, bei denen wir zunächst am meisten stutzig wären. Jener besitzt zwar zwei orale Scheitelbalken, jedoch keine Querverbindungen, und das Verhältnis $\frac{\text { Sch. }}{A \text {. }}$ ist bei beiden Skeletthälften gleich oder fast $=\frac{1}{1}$. Der Pluteus 35 hat außer den wenigen Querverbindungen und einer dreiwurzeligen Analarmstutze keine Merkmale, die auf einen für kleinkernige Larven ungewöhnlich starken Einfluß des muitterlichen Erbanteils hinweisen würden, denn das Verhältnis $\frac{\text { Sch. }}{\text { A. }}=\frac{1}{1}$ nnd die schwach ausgebildeten Sonderformen am Ende der analen Scheitelbalken lassen immerhin einen starken väterlichen Einfluß erkennen.

Diese kurze Erörterung zeigt, daß das Skelett der klein- und großkernigen Larven nicht nur im allgemeinen, sondern auch im einzelnen merkliche und deutlich kennzeichnende Unterschiede aufweist.

D. Scheinbare Abweichungen.

DaB Larven, die sich parthenogenetisch entwickelt haben, kleine Kerne haben können und die Merkmale der Mutter besitzen, liegt auf der Hand. Unter den vielen Hunderten von Larven, die ich betrachtete, fand ich eine, deren Skelett durchaus wie das von Sphaer- 
echinus gebildet war (Fig. 21) und die kleine Kerne besaß. Es geht nicht an, diese Larve mit den andern kleinkernigen Larven zu vergleichen und in Gegensatz zu stellen: der Unterschied ist dafür zu groß und das Auftreten einer solchen Larve zu selten. Wir sind auch nicht in Verlegenheit, diese scheinbare Ausnahme zu erklären. Wie ich oben schon mitteilte und später noch genauer angeben werde, waren vor der Befruchtung manche Eier in parthenogenetische Entwicklung eingetreten. Es liegt darum nahe, diese kleinkernigen Larven mit dem Sphaerechinus-Skelett als einen parthenogenetischen Pluteus aufzufassen.

Der Pluteus 10 der zweiten Tabelle ist eine Zwerglarve, wie aus den angegebenen Zahlen hervorgeht. Die Gestalt (Fig. 22, bei stärkerer Vergrößernng gezeichnet!) ist fast die der SphaerechinusLarve, aber die linke Analarmstitze ist vierkantig, die oralen Armstutzen sind sehr kurz, wie auch der rechte Oralscheitelbalken nur halb ausgebildet ist. Besonders hervorzuheben ist, daB dieser Pluteus weitaus die größten Kerne unter allen Larven besaß, die mir zu Gesicht kamen. Vielleicht kann man sich die Entstehung dieser Larve so denken: Durch mehrfache Monasterbildung ist das Chromatin des Eikerns vor der Zweiteilung sehr vermehrt and damit dieser selbst stark vergrößert worden; auf der Zweizellenstufe haben sich die beiden Zellen getrennt, und aus der einen ging die Zwerglarve hervor. Daß solche Trennungen von Furchungszellen mit vergrößerten Kernen vorkommen, konnte ich zweimal auf Schnitten sehen (allerdings war in beiden Fällen die Trennung noch nicht vollständig erfolgt), und daß aus einer der zwei ersten Furchungszellen eine wohl ausgebildete, doch entsprechend kleinere Larve entstehen kann, hat Driesch gezeigt.

Um nun auch eine klare bildliche Anschauung von der Verschiebung der Vererbungsrichtung zu geben, habe ich eine Reihe von Abbildungen hergestellt, die mit der Darstellung einer Strongylocentrotus-Larve beginnt und mit der einer Sphaerechinus-Larve schließt und so einen sinnfälligen Eindruck von dem stärkeren Hervortreten der mütterlichen Merkmale bei den großkernigen Larven vermittelt (Fig. 1-20).

An dieser Stelle möchte ich einen sonderbaren Einwand erwähnen, den kürzlich MaCbride (11) gegen die von Herbst bei der Kreuzung $\frac{S t r . O^{\top}}{S p h . Q}$ nachgewiesene Verschiebung der Vererbungsrichtung nach der mutterlichen Seite vorbrachte. „Thus Herbst, who studied 
chiefly the cross between Sphaerechinus and Strongylocentrotus, attaches great importance to the extent to which lattice-work appears in the skeleton of the arms of the hybrid. In the normal larva of Strongylocentrotus, it is true, all four arms are supported by unbranched calcareous rods, whilst in the normal larva of Sphaerechinus, each of the two posterior arms is supported by parallel rods connected by cross-pieces like the steps of a ladder, an arrangement which is termed, lattice-work ${ }^{6}$. But HERBST fails to take into account the fact that in the normal larva of Strongylocentrotus a lattice-work skeleton can appear as a variation, and hence an attempt such as he makes to estimate quantitatively the influence of one parent by the amount of lattice-work which appears in the hybrid rests upon an insecure foundation.* Da somit MACBRIDF nicht sieht, auf wie sicherem Grande die Feststellung der tatsächlich verschobenen Vererbungsrichtung ruht, möchte ich zeigen, daß der Fehler bei ihm liegt. MaCBRIDE gibt an, daß bei Strongylocentrotus-Larven auch Gitterstäbe vorkommen. Das stimmt ja wohl. StEInвRück (02) hat eine solche Analarmstïtze (Taf. I Fig. 24) abgebildet; doch besitzt diese nur eine Wurzel und zwei Querverbindungen. Solche Larven dürften aber so selten sein (HERBst z. B. hat bis jetzt nur vier derartige Strongylocentrotus-Larven gesehen) und so wenig als »normal < bezeichnet werden, wie eine menschliche Hand mit sechs Fingern normal genannt werden kann. Was kann das seltene Vorkommen aber bedeuten gegenuiber der ungeheuren Menge von wirklich normalen Strongylocentrotus-Larven? Nichts! Und daß in den Versuchen von HERBST gerade solche Strongylocentrotus-Samenfïden zur Befruchtung gelangt sein sollten, die auch ohne die vorhergehende Behandlung der Sphaerechinus-Eier die Ausbildung eines Gitterwerks bewirkt bätten, wird auch M.ACBRIDE im Ernst nicht annehmen. Wie wären denn die Larven dieses Versuchs, die kleine Kerne und meist nicht die Spur eines Gitters besaßen, zu erklären? Hier wäre wohl zufällig nicht allein der mütterliche, sondern auch der die Gitterbildung bewirkende Erbanteil der merkwlirdig *normalen « Väter ohne Einfluß geblieben?!

HeRBsT hat aber außer den Gitterstäben auch noch die Zabl der Wurzeln, die oralen Scheitelbalken, die Gestalt der analen Scheitelbalken, das Verhältnis $\frac{\text { Sch. }}{A .}$ und anderes in seine Betrachtungen und Rechnungen einbezogen und erst anf Grund all dieser Vergleiche seinen Schluß gezogen, der ja auch durch seine cytologischen Untersuchungen einleuchtend gemacht wurde. 


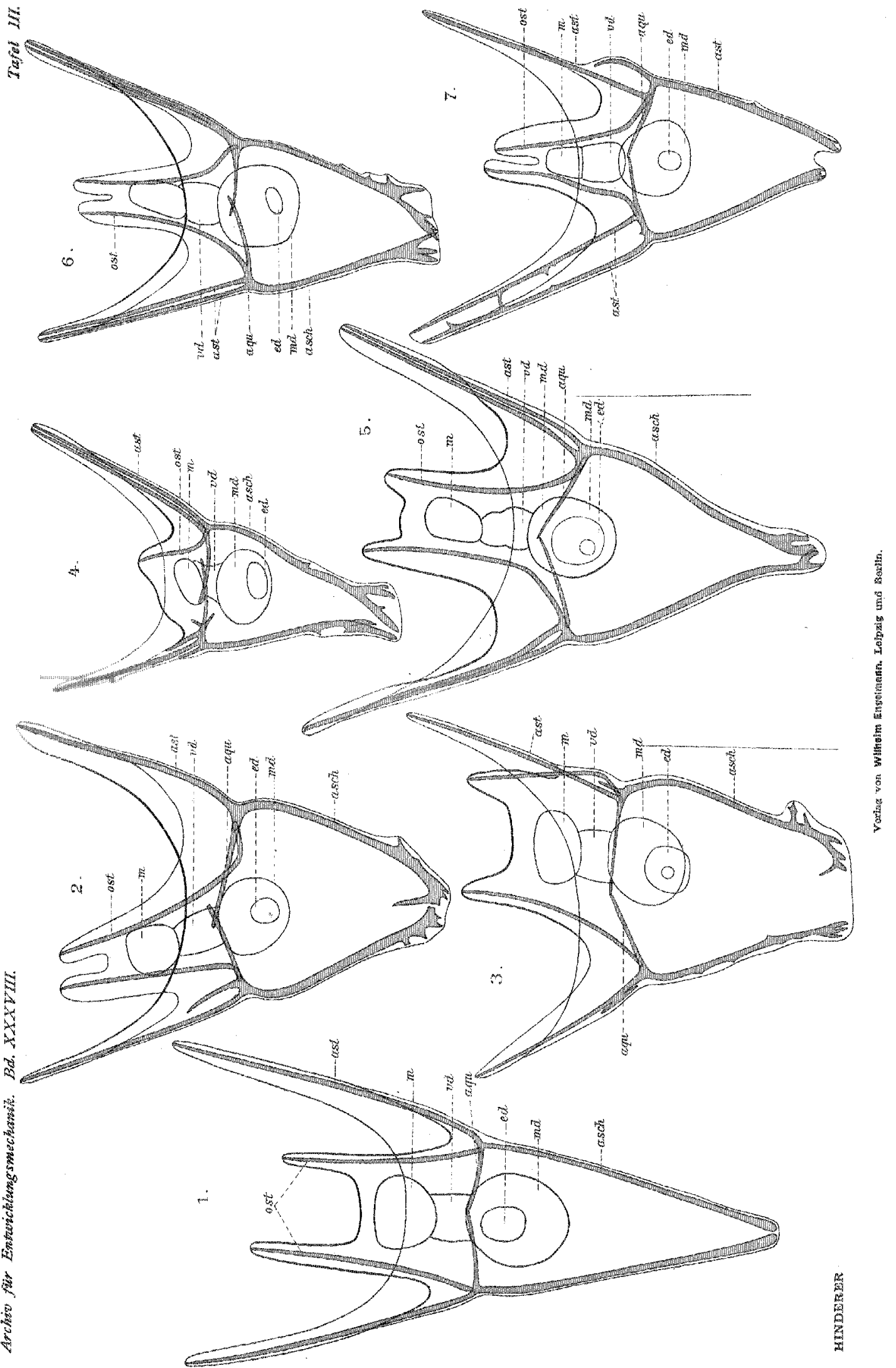




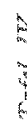
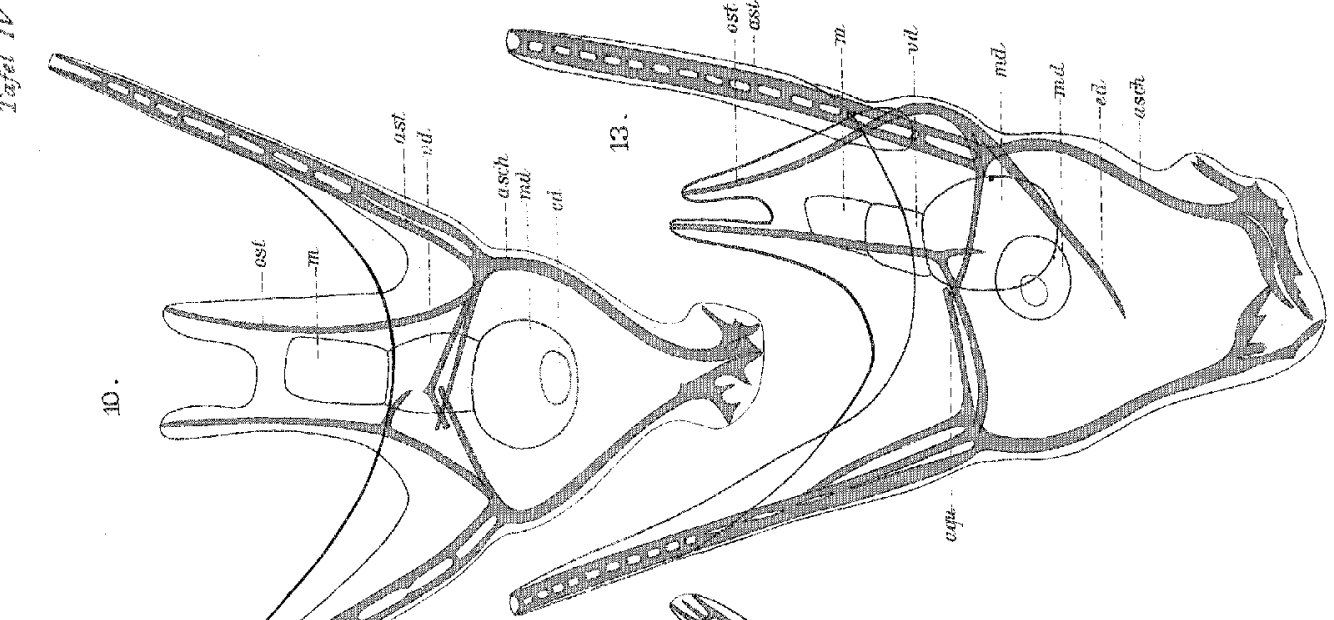

(1)

s

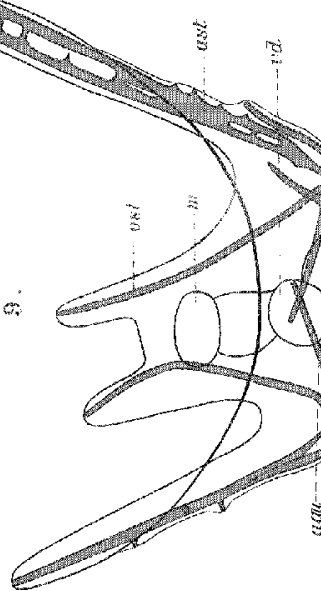

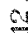
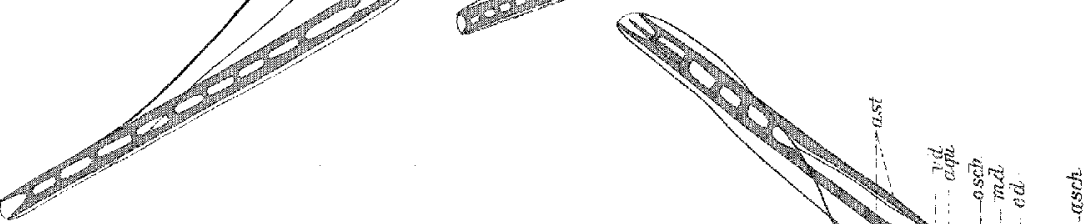


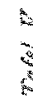

से

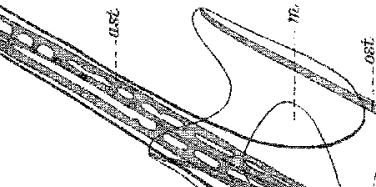

$\vdots$

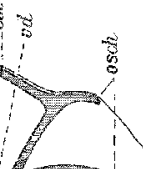

(1)

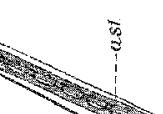
$\overleftrightarrow{8}$

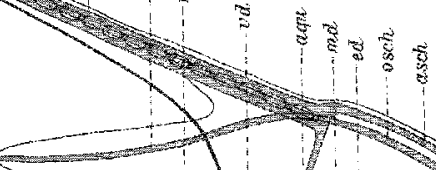

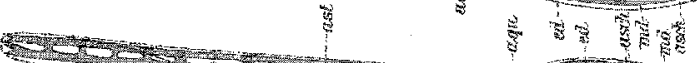
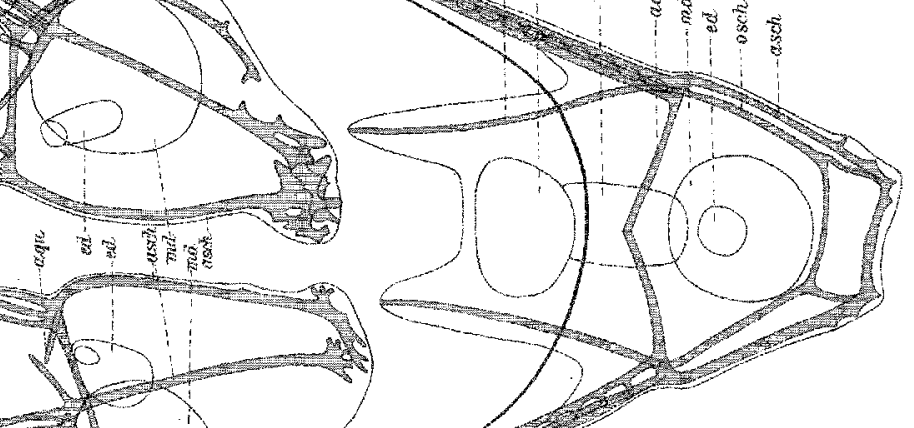

$\Leftrightarrow$

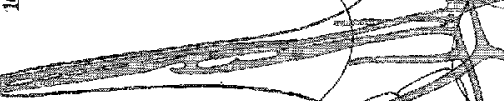

政
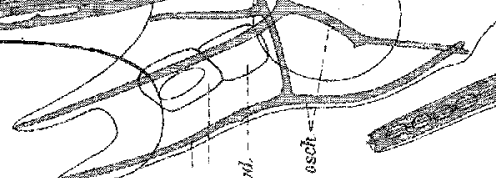

宊

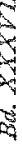

(4. 4,1 6.8. कै

10

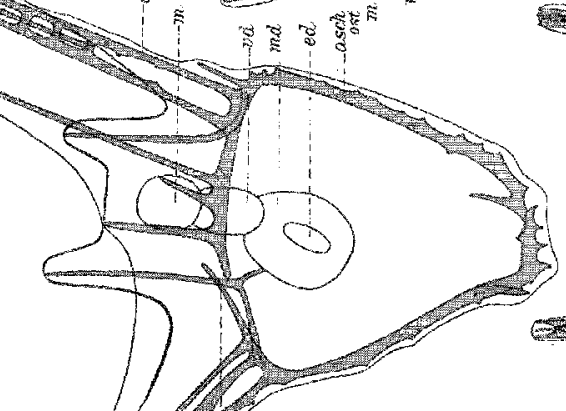

等 寒密
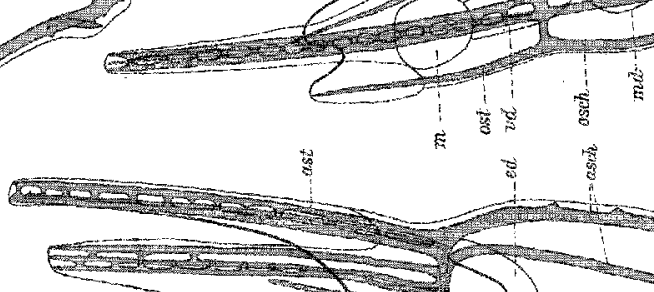

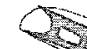

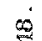

क्ष

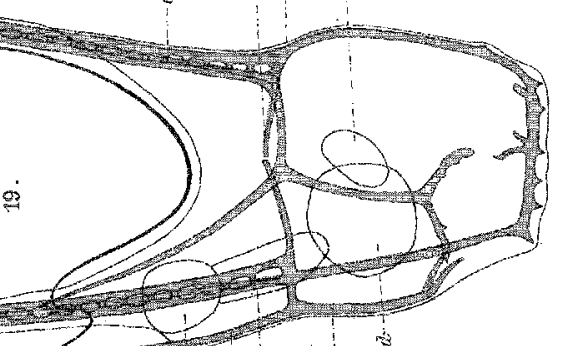


Der Einwurf MacbRides erscheint mir ebenso berechtigt wie etwa der: es sei nicht beweisend, daB der mutterliche Erbanteil ausschlaggebend geworden sei, wenn die Kinder einer Mutter mit sechsfingerigen Händen und eines Vaters mit gewöhnlicher Fingerzahl je sechs Finger an jeder Haud besäßen - denn es gebe auch Männer mit sechs Fingern an jeder Hand!

(Schluß folgt.)

\section{Erklärung der Abbildungen.}

Buchstabenbezeichnungen.

$\begin{array}{llll}\text { aque analer Querstab, } & m d & \text { Mitteldarm, } \\ \text { asch } & \text { analer Scheitelbalken, } & \text { osch } & \text { oraler Scheitelbalken, } \\ \text { ast } & \text { Analarmstiitzen, } & \text { ost } & \text { Oralarmstiitzen, } \\ \text { ed } & \text { Enddarm, } & v d & \text { Vorderdarm. } \\ m & \text { Mund, } & & \end{array}$

\section{Tafel III-V,}

Fig. 1-20. Plıtei, gezeichnet bei der Vergrößerung Seiber T Obj. 3, Ok. 3, und bei eingeschobenem 'Tubus. Für die Längen sind nur die Angaben in den Tabellen 1 und 2, nicht aber die Zeichnungen maßgebend.

Fig. 1. Strongylocentrotus-Pluteus aus reiner Zucht.

Fig. 2-9. Kleinkernige Plutei aus der Zucht $\frac{\text { Strongylocentrotus }}{\text { Sphaerechinus } \text { 우 }}$

Fig. 10-19. Großkernige Plutei aus der Zucht $\frac{S t r . ~}{\text { Sph. }}$.

Fig. 20. Sphaerechinus-Pluteus aus reiner Zucht. 